abilities of cerebral function forced as well as possible. Some cases of expanding craniectomy showed excellent effects against our expectations several years later.

\title{
i-14. The Treatment for Lumbo-sacral Meningocele and Meningomyelocele in Children
}

\author{
Shuzo Okumura, Hideo Nobuto and Kousuke Ohta \\ Neurosurgical Service, National Hospital in Okayama \\ Itsuro YAMANOUCHI \\ Pediatric Service, National Hospital in Okayama
}

The experience of 15 new born infant having lumbo-sacral meningocele, possibly including meningomyelocele, in the latest 4 years is reported.

2 cases, out of 7 cases those have had conservative treatment, are markedly improved in the following 3 years with scarforming of the meningeal cyst and without gross neurological defect. One case, out of 7 conservative case, is growing with arrested moderate hydrocephalus. 4 cases died by 4 months due to infection and sepsis.

3 cases those have had plastic operation on the meningeal cyst without shunt operation of the CSF, died in following 4 months due to intracranial hypertension and marasmus.

5 cases those have had ventriculo-atrial shunt of CSF in the second month after the birth are presenting satisfactory growth without gross neurological defect not requiring further surgical procedure except one case which had plastic operation for the meningeal cyst because spontaneous size reduction was not observed.

\section{i-15. Subdural Collection in Infancy}

\author{
Associated with Congenital Anomaly of the Brain \\ Tsutomu Iwakuma and Kazuhiko Iwashita \\ Neurosurgical Department, St. Luke's International Hospital
}

Five cases of subdural collection in infancy were experienced, ( 3 males and 2 females). Two out of five cases were associated with congenital anomalies of the brain.

Case 1: 5 months old male infant with $2,000 \mathrm{~g}$ in birth weight, 39 weeks in gestation, was admitted because of convulsion and enlarged head. Physical examination disclosed generalized developmental retardation. Funduscopic ex- 
amination revealed optic nerve atrophy. Subdural taps showed bilateral accumulations of bloody fluid.

X-ray examination showed abnormal radiolucent line on the parietal area of the skull. Porencephaly also found by P.E.G. A large avascular zone with carotid angiography. Craniotomy for removal of encapsulated pathologic membrane was carried out. Four months later reaccumulation of the fluid was noted. So the result of craniotomy has been discouraged.

Case 2: 3 months old female infant with normal delivery, $2,250 \mathrm{~g}$ in birth weight, 41 weeks in gestation, was admitted because of poor feeding. There was a developmental retardation. Funduscopic examination revealed optic nerve atrophy. Subdural taps showed bilateral accumulation of xanthochromic fluid containing $800 \mathrm{mg} / \mathrm{dl}$ of protein.

Plain skull $\mathrm{x}$-ray also showed abnormal radiolucent lines. P.E.G. showed porencephaly in the parietal region. Carotid angiography showed large avascular zone without all sinuses. Exploratory burr holes were made. There was a encapsulated outer membrane, but no inner membrane. Underlying brain was severely atrophic, hard in consistence and no pulsation. The falx could not found. The result of operation was poor because no expansion of the brain has been noted.

We reported 2 cases of subdural collections with marked brain atrophy, porencephaly and lack of falx.

Both of these cases were low birth weight infants and full term infants. The origin of these subdural collection is unknown but they seemed to occure intrauterine. Their prognosis was poor.

\section{Discussion to i-15.}

\section{Tomio Ohta, Shuro Nishimura, Tamotsu Asakura, Ikuo Ishikawa and Atsushi KaWARAZAKI \\ Department of Neurosurgery, Osaka City University Medical School}

Two cases of hydrocephalic infants combined with unilateral subdural fluid collection have been presented. Case 1. One and half year-old boy. No abnormality of the pregnancy, delivery and head circumference at birth have been noted. Since his 3 month old age gradual increase of head circumference, and since his 9 month old age slight hemiparesis on the left side have been found. At the time of admission, head circumference was $56 \mathrm{~cm}$ with remarkable protrusion in the left frontal region, and positive transillumination was demonstrated on the left side of the skull vault. Left hemiparesis has been noted with hyperreflexia and Babinski sign. Downward displacement of all branches of cerebral vessels and wide spread avascular space have been revealed in the left carotid angiography. Craniotomy was performed on the side of subdural fluid collection 